\title{
Réflexion sur l'autonomie de la morphologie linguistique
}

\author{
Samuel Tuesday Owoeye (Ota, Nigeria)
}

\begin{abstract}
Sous les auspices de la grammaire traditionnelle, l'étude des mots que l'on appelle contemporainement la morphologie jouissait incontestablement d'analyse autonome. Or, depuis l'inauguration de la linguistique moderne issue du structuralisme saussurien, le sort de la morphologie n'a cessé de subir des suppressions réductionnistes. Par conséquent, la morphologie est aujourd'hui traitée de deux perspectives plus ou moins opposées: analyse autonome versus analyse dépendante. Après avoir survolé les instanciations linguistiques cherchant à établir le statut scientifique de la morphologie l'article confirme modestement le statut autonome scientifique de la morphologie et postule également qu'aucune branche de la linguistique, la morphologie y comprise, ne jouit d'un statut autonome total.
\end{abstract}

\section{$1 \quad$ Introduction}

La morphologie est traditionnellement définie comme le domaine de la linguistique qui étudie la combinaison séquentielle des morphèmes pour former des mots acceptables dans une langue naturelle donnée. Bien que cette définition semble assez cerner ce champ d'étude linguistique, la théorisation et l'analyse des faits morphologiques ont connu beaucoup de perspectives et d'orientations, tantôt opposées tantôt complémentaires, depuis la découverte de la grammaire paninienne du sanskrit. Même s'il est vrai qu'aucun des domaines de la linguistique n'est sans des considérations débattues, les débats morphologiques sont plus controversés surtout quand il s'agit de son statut linguistique. Pendant une longue période, les linguistes, du structuralisme au générativisme, supprimaient l'autonomie de la morphologie. Cette suppression, qui a poussé Anderson (1982) de titrer son article "Where is Morphology", "est généralement décrite par Kerleroux (2006: 2313) lorsqu'il remarque:

La morphologie a connu des bouleversements plus considérables que d'autres domaines constitutifs de la grammaire. Pour certains, l'événement majeur a été la fondation, puis la détermination de programmes de recherche successifs. Ainsi peut-on parler de la fondation de la phonologie, avec Troubetzkoy et Jakobson à la fin des années 1920. Ou de la naissance de la syntaxe en 1957 avec Structures syntaxiques, où Chomsky fixait l'objectif nouveau de rendre compte de toutes les phrases bien formées d'une langue. Mais la morphologie, comme étude de la structure des mots, après avoir été traitée comme la composante principale de la grammaire, a totalement disparu du panorama grammatical, absorbée qu'elle fut dans les deux territoires limitrophes de la syntaxe et de la phonologie.

A part le problème de fondation noté ci-dessus, l'ambition des linguistes d'intégrer la morphologie dans les territoires limitrophes (surtout dans la syntaxe) a été renforcée par un autre facteur pertinent. Les deux domaines (morphologie et syntaxe) concernent chacune la première articulation en ternes martinetiens. Pour expliquer ce facteur, nous citons Fradin (1994:1-2) à l'appui:

Les règles morphologiques s'occupent $\mathrm{du}$ rapport entre la face matérielle des signes linguistiques (le substrat) et leur contenu (dénommé ici l'abstrat). Il en va de même des règles ou mécanismes syntaxiques puisque les unités syntaxiques présentent elles aussi une face 
substrat et une face abstrat. Cette situation contraste avec celle de la phonologie qui n'est concernée que par la deuxième articulation. Les règles phonologiques mettent en rapport le substrat avec le substrat (rapport son X son).

Si la morphologie et la syntaxe se situent dans la première articulation, des méthodes d'analyse similaires doivent s'appliquer à elles et par conséquent l'inévitabilité d'une concurrence d'analyse. Dans cette concurrence naturelle, toutefois, la syntaxe semble en être la plus susceptible d'intégrer la morphologique parce qu'il y a des faits morphologiques qui sont régis par la syntaxe: notons ici que les règles flexionnelles dépendent largement de règles syntaxiques. Au contraire, la phonologie, même avec l'existence du domaine de la morphophonologie, ne peut jamais envisager une telle ambition. Les faits morphophonologiques ne sont que des phénomènes d'interface nés des contraintes phonotactiques des langues naturelles. La morphophonologie n'est qu'un domaine collaboratif car aucune concurrence d'analyse ne peut exister entre la morphologie et la phonologie. Compte tenu de ce sort caractéristique de la morphologie, une problématique s'impose toujours: la morphologie, est-elle un domaine linguistique autonome ? Cette question est un objet de débat qui vit toujours. Dans cet article, nous confirmons l'autonomie de la morphologie. Nous postulons que la morphologie est un domaine linguistique scientifiquement autonome dans la mesure où elle étudie la structure interne des mots. Cependant, tout comme les autres domaines formels de la linguistique, elle ne jouit pas d'autonomie absolue étant donné le caractère social des langues naturelles.

\section{La morphologie à la recherche de l'autonomie}

On peut reconnaître quatre phases dans le développement théorique de la morphologie. Saussure (1995: 15), avec qui s'ouvre la linguistique structurale avait d'abord noté que la linguistique "a passé par trois phases successives avant de reconnaître quel est son véritable et unique objet." Il les identifie respectivement à la grammaire traditionnelle, la philologie (linguistique historique) et au structuralisme. Il s'ajoute à ces trois phases une quatrième, la linguistique générative chomskyenne. Au commencement de la grammaire traditionnelle qui remonte au cinquième siècle avant $\mathrm{J}-\mathrm{C}$, le mot est considéré "le signe linguistique minimal" (Beard 1995: 1) et donc la structure des mots ne constitue pas un objet d'étude spécifique (Asher \& Simpson 1994). Cependant, pendant la période de la linguistique historique qui est une continuation de la culture normative de la grammaire traditionnelle et représentée par les linguistes tels que Franz Bopp (1791-1867), Williams Jones (1746 1794), Jacob Grimm (1785 -1863), August Schleicher (1821 - 1868), Wilhelm von Humboldt (1767 - 1835), et Jan Baudouin de Courtenay (1845 - 1929), l'étude de la structure des mots devient un objet d'étude autonome. Parlant alors de la grammaire traditionnelle, la morphologie est un composant principal jouissant d'un statut autonome à coté de la syntaxe. Du point de vue de la théorie traditionnelle des langues, l'étude de la morphologie se subdivise en deux types majeurs: la morphologie dérivationnelle et la morphologie flexionnelle. Tandis que la dérivation s'occupe de la formation des mots nouveaux à partir des mots de base par l'ajout des affixes dérivationnels, la morphologie flexionnelle étudie la combinaison des désinences grammaticales qui changent la personne grammaticale, le nombre ou le genre des mots de base. Ces affixes et désinences grammaticales sont des morphèmes. En tant que composante significative de la grammaire traditionnelle, la morphologie de morphèmes jouit d'une hégémonie grammaticale pendant des siècles. Or vers la fin du XIX $\mathrm{XI}^{\mathrm{e}}$ siècle, cette hégémonie commence à céder la place aux études de langues plus scientifiques et plus descriptives avec le lancement de la linguistique structurale par Ferdinand de Saussure avec son Cours de linguistique générale publié pour la première fois en 1916. 
Saussure (1995: 186) n'a pas hésité en affirmant que "linguistiquement, la morphologie n'a pas d'objet réel autonome; elle ne peut constituer une discipline distincte de la syntaxe." Suivant les pas de Saussure, les théories linguistiques héritières du structuralisme (le distributionnalisme, le fonctionnalisme, la grammaire tagmémique etc), de l'Europe à l'Amérique, n'accordaient la morphologie ni une position privilégiée ni un statut autonome comme elle était traitée au sein de la syntaxe. Ce scénario est confirmé par les propos de Harris (1951: 58) quand il dit que "les résultats en syntaxe et en morphologie sont obtenus par les mêmes procédures, si bien qu'aucune distinction n'est tracée entre eux". Avec la linguistique structurale et les théories linguistiques collaboratrices, tous les composants de la grammaire y compris la morphologie sont soumises aux études scientifiques, délaissant l'approche normative qui caractérise la grammaire traditionnelle. L'approche traditionnelle qui est plus ou moins réglementée devient un objet de critique par Saussure (1995: 13). Selon lui,

Cette étude, inaugurée par les Grecs, continuée principalement par les Français, est fondée sur la logique et dépourvue de toute vue scientifique et désintéressée sur la langue elle-même ; elle vise uniquement à donner des règles pour distinguer les formes correctes des formes incorrectes ; c'est une discipline normative, fort éloignée de la pure observation et dont le point de vue est forcément étroit.

Bien que le structuralisme saussurien ne présente pas directement les faits de la morphologie, il montre que tout signe linguistique entre dans deux sortes de rapports que Saussure appelle les rapports syntagmatiques et les rapports associatifs. Les rapports syntagmatiques sont ceux que, dans le discours, les mots contractent entre eux, en vertu de leur enchaînement. Ils sont fondés sur le caractère linéaire de la langue, qui exclut la possibilité de prononcer deux éléments à la fois et contraint par conséquent les unités linguistiques à se présenter les unes après les autres dans la chaîne parlée.

Les rapports associatifs se définissent quant à eux par le fait que, en dehors du discours, les mots s'associent dans la mémoire du locuteur et de l'auditeur. Signalons que les deux séries de rapports (rapports paradigmatiques et rapports syntagmatiques) qui caractérisent les langues naturelles impliquent la morphologie. Pour reprendre un des exemples de Ferdinand de Saussure, le mot enseignement est impliqué dans une série associative qui a en commun le radical enseign-, à savoir enseignement, enseigner, enseignons, renseigner, etc., et dans une autre série associative qui a en commun le suffixe -ment, à savoir enseignement, changement, armement, etc. Ces deux séries associatives ont, chacune, une unité significative, c'est-à-dire une unité ayant une forme et un sens. Faisons remarquer également que le même mot enseignement est aussi impliqué dans une série associative où l'élément commun est seulement phonétique - c'est-à-dire le son [ã], à savoir: enseignement, clément, justement, etc., et dans une série associative qui repose sur la seule analogie des signifiés, à savoir enseignement, instruction, apprentissage, éducation. Ferdinand de Saussure (1995: 97) oppose clairement les rapports associatifs et les rapports syntagmatiques en les caractérisant de la façon suivante: "le rapport syntagmatique est in praesentia: il repose sur deux ou plusieurs termes également présents dans une série effective. Au contraire, le rapport associatif unit des termes in absentia."

Si les thèses génératives initiales de Chomsky marquent une rupture remarquable avec le structuralisme, elles n'ont pas rompu la marginalisation théorique de la morphologie. En faisant de la syntaxe un domaine-roi de la linguistique, Chomsky (1957: 52) relègue la morphologie en ces termes:

Nous construisons une dérivation élargie commençant par Phrase. En passant par les règles [de structure syntagmatique], nous construisons une séquence terminale qui sera une suite de morphèmes, pas nécessairement dans l'ordre correct. Nous passons ensuite par une suite de transformations [...], qui peuvent réordonner les séquences, ajouter ou effacer les morphèmes. 
Elles ont pour résultat la production d'une séquence de mots. Nous passons alors par les règles morphophonologiques qui convertissent cette séquence de mots en une séquence de phonèmes.

Pour Chomsky, donc, les faits de la morphologie dérivationnelle ainsi que ceux de la morphologie flexionnelle sont analysables au sein de la syntaxe. Son propos peut se résumer ainsi: les mots dérivés tels responsabilité, construction, abattage, etc. sont analysables comme des résultats d'une opération de transformation phrastique. Par exemple, la phrase la construction de la maison a été faite par l'ingénieur nigérian est une phrase dérivée d'une autre phrase de base l'ingénieur nigérian a construit la maison. Ainsi, on peut rendre compte de la construction du mot-nom "construction" à partir du mot-verbe "construire" au sein de la syntaxe. Il est bien évident, alors, que du structuralisme saussurien jusqu'aux premières thèses générativistes de Chomsky, la morphologie était traitée comme un sous-domaine de la syntaxe. Cependant, l'élaboration de l'hypothèse lexicaliste de Chomsky (1970) marque le retour de la morphologie autonome.

L'hypothèse est née de l'observation qu'il s'avère impossible de construire les mots complexes dérivationnels qui présentent des distributions irrégulières et des lacunes au moyen de l'opération de transformations syntaxiques. Chomsky observe que les mots dérivés construits à partir des transformations nominales déverbalisées restent invisibles pour la syntaxe car on peut également avoir des constructions syntaxiques similaires qui ne sont pas engendrées par l'opération de transformation déverbale. Soit les deux phrases suivantes:

a. La construction de la maison a été faite par l'ingénieur nigérian.

b. L'auteur du livre a été arrêté par la police.

Dans les deux phrases, les syntagmes nominaux la construction de la maison et l'auteur du livre ont la même structure de type $\mathrm{SN}_{1}+$ Prép $+\mathrm{SN}_{2}$. Cependant, seule la première phrase contient un mot dérivé "construction". S'il en est ainsi, les règles syntagmatiques restent inadéquates pour rendre compte de la construction des mots dérivés car tout ce que voit la syntaxe dans le mot "construction" est sa catégorie grammaticale $N$ mais pas le suffixe -ion. Grâce à cette hypothèse, donc, une composante lexicale, comportant des règles de construction autonomes, était introduite à côté de la composante syntaxique et par conséquent, une démarcation est née entre la grammaire des mots et la grammaire des phrases, pour reprendre les termes de Bach (1983), au sein de la grammaire générative. Parmi les défenseurs du statut autonome de la morphologie dérivationnelle lancée par Chomsky, on peut citer, à titre d'exemple, Dell (1970), Halle (1973), Aronoff (1976), Selkirk (1982), et Scalise (1984).

Avec le lancement de l'hypothèse lexicaliste, la morphologie devient de plus en plus indépendante de la syntaxe. Depuis lors, la morphologie n'a cessé de connaître des épanouissements qui ont fait d'elle un domaine très important de la linguistique. Cette importance est confirmée par Spencer et Zwicky (1998: 8) lorsqu'ils disent:

Morphology is at the conceptual centre of linguistics. This is not because it is the dominant subdiscipline, but because morphology is the study of word structure and words are at the interface of phonology, syntax and semantics.

Selkirk (1982, cité et traduit par Kerleroux 2001), bien qu'il revendique l'autonomie de la morphologie, estime cependant que cette autonomie n'est pas absolue dans la mesure où elle se limite aux unités concaténatives, c'est-à-dire les affixes, que la syntaxe ne voit pas. Il défend sa thèse en ces propos:

Il semble possible de fait de construire la structure des mots comme un système autonome. Selon moi, la catégorie Mot se trouve à l'interface dans la représentation syntaxique de deux variétés de structures, qui doivent être définies par deux ensembles distincts de principes dans la grammaire. Cependant, je vais défendre la thèse que la structure des mots a les mêmes 
propriétés formelles générales que la structure syntaxique, et, en outre, qu'elle est générée par le même genre de système de règles. Pour souligner cette similarité fondamentale, j'emploierai les termes W-syntaxe, et W-syntaxique, plutôt que morphologie et morphologique, pour parler de la structure des mots. (Selkirk, 1982: 2, cité par Kerleroux 2001: 17)

La thèse selkirkienne, en remplaçant les termes "morphologie" et "morphologique" en "Wsyntaxe" et "W-syntaxique" respectivement paraît un peut déroutante. On estimerait que toute revendication de l'autonomie de la morphologie est censée retenir les termes ici délaissées par Selkirk. Il semble que la position de Selkirk est entre ceux revendiquant l'autonomie de la morphologie et ceux qui la considèrent comme une sous-composante de la syntaxe. En dépit de l'argument de Selkirk, la thèse qui semble être communément acceptée par les morphologues néo-chomskyens est celle qui pose l'existence de plusieurs opérations morphologiques dont la syntaxe ne peut pas rendre compte. Par conséquent, des règles proprement morphologiques doivent être postulées pour rendre compte de telles opérations. Tandis que la morphologie flexionnelle se laisse régir partiellement par les règles syntaxiques, la morphologie dérivationnelle (ou constructionnelle) se régit par les règles proprement morphologiques. Suite à cet essor morphologique, ce que Aronoff (1976: 4) et Mortureux (2004: 37) appellent respectivement "la renaissance de la morphologie" et "the return of Morphology", les termes tels que 'Règles de Construction de Lexèmes' (RCLs), 'Règles de Construction de Mots' (RCMs), Règles de Formation de Lexèmes' (RFLs), 'Règles de Formation de Mots' (RFM) furent nés. Les RCLS sous-tendent la construction des lexèmes dérivés à partir des lexèmes simples et résultent des efforts des morphologues néo-génératifs voulant séparer la morphologie de la syntaxe. Les premières RCLs sont postulées par Aronoff (1976), adaptant les Règles de Réécriture (RR) chomskyennes. Par exemple, Aronoff (1976: 86) donne une RCL des dérivés agentifs anglais en -er comme suit:

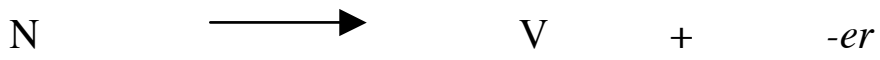

Pour les dérivés agentifs français en -eur, on aura quelque chose comme suit:

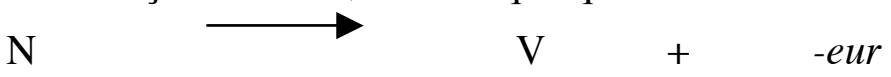

Depuis Aronoff, cependant, les RCLs ont subi des reformulations plus fines. A titre d'exemple, nous reproduisons ici une RCL pour les dérivés agentifs français en -eur, proposée par Fradin (2003: 254):

\begin{tabular}{|c|c|c|c|c|}
\hline & $\mathrm{I}$ & INPUT & $\mathrm{O}$ & OUTPUT \\
\hline$(\mathrm{F})$ & $\mathrm{a}$ & $(\ldots)$ & $\mathrm{a}$ & (...œr) \\
\hline \multirow[t]{3}{*}{$(\mathrm{SX})$} & $\mathrm{b}$ & cat: v & $\mathrm{b}$ & cat: $n$ \\
\hline & $\mathrm{c}$ & str-arg $<\mathrm{SN} 0 \ldots>$ & $\mathrm{c}$ & \\
\hline & $\mathrm{d}$ & rôle: SNO = agent & $\mathrm{d}$ & \\
\hline$(\mathrm{S})$ & $\mathrm{e}$ & $\left(\lambda e x . \lambda \operatorname{ev} .\left(\mathrm{V}^{\prime} \bullet \operatorname{ev} \bullet x \ldots\right)\right)$ & e & $\left(\lambda \mathrm{V}^{\prime} . \lambda \mathrm{x} . \exists \mathrm{ev} .\left(\mathrm{V}^{\prime} \bullet \mathrm{ev} \bullet \mathrm{x} \ldots\right) \wedge\right.$ activité-sociale' $\left.\bullet \mathrm{ev}\right)$ \\
\hline
\end{tabular}

$\mathrm{Vu}$ à travers ce tableau, la représentation des règles morphologiques de l'optique lexématique est multidimensionnelle. La rubrique $\mathrm{F}$ fournit des informations sur la forme phonologique du lexème base ainsi que le lexème construit. Dans le même ordre, $\mathrm{SX}$ et $\mathrm{S}$ représentent les propriétés syntaxiques et sémantiques respectivement. Les informations fournies par la rubrique SX comprennent la catégorie lexicale des deux lexèmes impliqués ainsi que la structure argumentale et le rôle syntaxique du lexème base. Les propriétés sémantiques dans la rubrique S sont exprimées sous forme de lambda calcul. Qualitativement, cette règle démontre que la construction d'un lexème nominal déverbalisé à partir du suffixe-eur exige un verbe de base qui a une structure argumentale agentive, c'est-à-dire que le verbe de base doit pouvoir avoir un agent pour qu'il puisse recevoir le suffixe -eur. 
Bien que l'hypothèse lexicaliste marque une révolution morphologique remarquable, elle aboutit à diviser les morphologues néo-génératifs en deux camps divergents. Le premier camp regroupe les morphologues qui préconisent une version faible de l'hypothèse (Weak Lexicalist Hypotheses) selon laquelle seule la morphologie dérivationnelle est indépendante des règles syntaxiques (voir Aronoff 1976; Anderson 1982; Corbin 1987). C'est cette version de l'hypothèse lexicaliste qui pousse Perlmutter (1988) à formuler l'hypothèse de la morphologie scindée (Split Morphology Hypotheses). Selon cette hypothèse, tandis que la morphologie dérivationnelle est pré-syntaxique, la morphologie flexionnelle se trouve après la syntaxe et se laisse régir par les opérations morphosyntaxiques; ce qui veut dire qu'il y a deux composants morphologiques dans la grammaire. Le deuxième camp rassemble les morphologues qui soutiennent la morphologie unitaire, la version forte de l'hypothèse lexicaliste (Strong Lexicalist Hypotheses), selon laquelle la morphologie flexionnelle est également considérée indépendante des opérations syntaxiques (voir Halle 1973; Lapointe 1981; Sciullo et Williams 1987). Même avec ses deux versions opposées, l'hypothèse lexicaliste est généralement considérée comme la théorie libératrice de la morphologie. Cependant, la libération n'allait pas être homogène car au début des années 90 , une autre approche à l'étude morphologique, appelée la Morphologie Distribuée, était inaugurée au sein de la grammaire générative.

La morphologie distribuée, énoncée par Morris Halle et Alec Marantz dans Halle \& Marantz $(1993 ; 1994)$, est une théorie anti-lexicaliste selon laquelle les opérations attribuées au lexique dans l'hypothèse lexicaliste et ses approches connexes sont distribuées entre différentes composants de la grammaire. Précisément, Halle \& Marantz (1993: 111-112) décrivent leur approche en ces termes:

We have called our approach Distributed Morphology (hereafter DM) to highlight the fact that the machinery of what traditionally has been called morphology is not concentrated in a single component of the grammar but rather is distributed among several different components.

Ainsi, cette théorie postule que la structure des mots, contrairement à l'approche lexicaliste, est tout d'abord manipulée par la syntaxe car des opérations syntaxiques combinent les nœuds terminaux pour créer des mots, et ce avant l'insertion lexicale. Il est clair que cette approche distribuée est une façon subtile de réintégrer la morphologie dans la syntaxe après un bref moment d'autonomie sous l'hypothèse lexicaliste. Cette réintégration a été subtilement faite par l'inclusion d'une nouvelle composante Structure Morphologique au schéma de la théorie syntaxique générative connue sous le nom de Principes et Paramètres (Chomsky 1981):

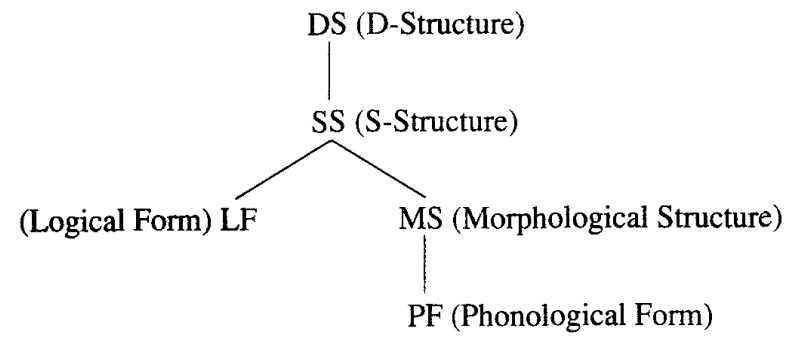

DS = Structure Profonde, SS = Structure de Surface, $\mathbf{L F}=$ Forme Logique, MS = Structure Morphologique, PF = Forme Phonologique (Schéma de la Morphologie Distribuée, tiré de Halle \& Marantz 1993: 114).

Pour expliquer le fonctionnement de la morphologie distribuée, ses promoteurs ont avancé trois principes qui sont l'insertion tardive (Late Insertion), la sous-spécification (Underspecification) et la structure syntaxique hiérarchisée de haut en bas (Syntactic Hierarchical Structure All the Way Down). Par le principe d'insertion tardive, les nœuds 
terminaux sont pris comme des complexes de traits sémantiques et syntaxiques abstraits qui sont manipulés au niveau de la structure morphologique. Par ce dernier principe, des items lexicaux sont produits et insérés dans les phrases. L'insertion de ces items lexicaux est tardive car elle ne se fait qu'après les manipulations morphosyntaxiques et avant les traits phonologiques qui déterminent la prononciation des dérivations syntaxiques ainsi produites par une opération d'Épellation (Spell-Out). Quant au principe de sous-spécification, il se définit par le fait qu'il n'est pas obligatoire qu'il y ait une correspondance parfaite entre les traits de l'item lexical et ceux du nœud terminal. Alors pour qu'un item lexical soit inséré dans un nœud terminal, les traits qui identifient ce même item doivent être un sous-ensemble de traits que possède le nœud terminal. Finalement, la structure syntaxique hiérarchisée de haut en bas veut dire que les nœuds terminaux où sont insérés les items lexicaux sont organisés d'une façon hiérarchisée et très essentiellement sous forme de structures déterminées par les opérations syntaxiques.

\section{Quelle sorte d'autonomie pour la morphologie?}

Ayant survolé le débat "autonomiste versus non-autonomiste" que subit la morphologie depuis le structuralisme saussurien jusqu'aujourd'hui, nous voulons souligner qu'aucune des deux dispositions n'est totalement à rejeter. Si notre position dans cette étude est de défendre un domaine linguistique autonome de la morphologie, ce serait erroné de prétendre que la morphologie est complètement indépendante de la syntaxe. En effet, du point de vue flexionnel, on ne peut pas séparer la morphologie de la syntaxe. Soit la phrase française suivante et sa représentation minimaliste:

$\begin{array}{llll}\text { Il } & \text { écrit } & \text { une } & \text { lettre } \\ \text { [3pers, sg, masc, nom] } & \text { [pré, ind, 3pers, sing, trs.] } & \text { [sg, fém ind] } & \text { [sg, fém acc] }\end{array}$

Simplement, la structure interne du mot-verbe écrit ainsi que celle du mot-déterminant une est déterminée par l'opération fusion de la syntaxe minimaliste. L'opération fusion, selon le Programme Minimaliste (MP) régit l'agencement des items lexicaux dans une phrase grammaticale. Si le syntagme nominal il dans la phrase ci-dessus est remplacé par un autre syntagme nominal nous, le mot-verbe écrit sera autre chose: écrivons. Aussi, si on remplace le syntagme nominal lettre par un autre syntagme nominal masculin dit livre, le déterminant une deviendra un. Ceci montre que la morphologie n'est pas totalement autonome. Mais cette dépendance tient pour les autres domaines de la linguistique. À vrai dire, aucune branche de la linguistique n'est totalement indépendante.

On a appris de Saussure qu'une langue est un système contenant des éléments interreliés. Du point de vue structurel, donc, on peut confirmer que la langue est structurée de la phonétique jusqu'à la sémantique. Ce rapport structurel peut être schématisé comme suit:

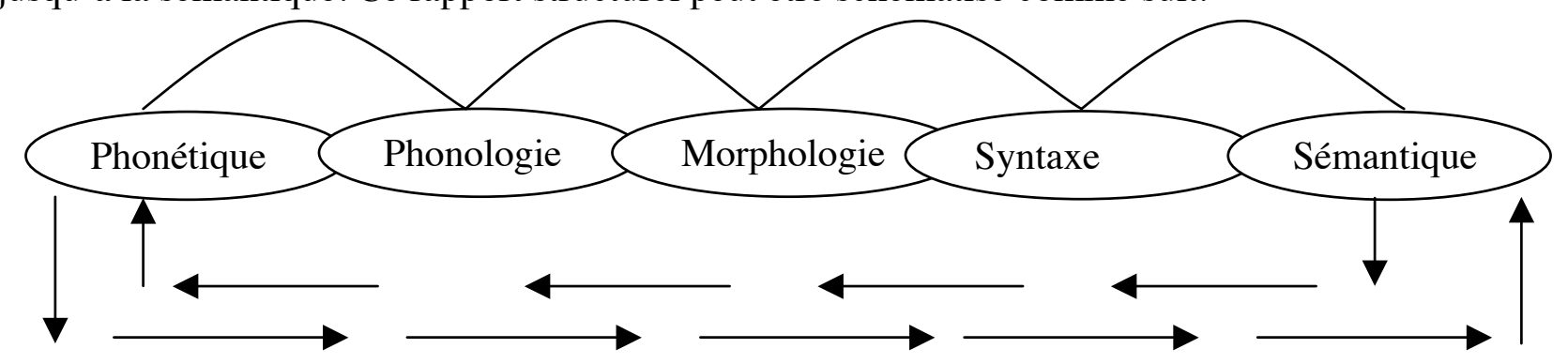

Le schéma ci-dessus montre que la langue est un macrosystème structurel relié par des microsystèmes ayant, eux aussi leurs structures chacun. Aucun de ces microsystèmes structurels - phonétique, phonologie, morphologie, syntaxe, sémantique - ne peut se 
proclamer totalement autonome des autres. S'il en est ainsi, le fait que le domaine flexionnel de la morphologie est gouverné par les règles syntaxiques ne peut constituer un argument susceptible à nier le statut autonome de la morphologie parmi les autres microsystèmes.

\section{Conclusion}

Notre tâche dans cette étude a été de mettre en relief le statut changeant de la morphologie dans les différentes approches théoriques. Si la construction des mots fléchis peut être abordée dans le microsystème syntaxique, la réflexion que nous venons de faire suggère que la construction dérivationnelle des mots peut être considérablement traitée hors de la syntaxe. Même si on peut faiblement arguer que la construction des mots dérivés nominaux tels que construction, triage, développement, possibilité, porteur, négociant, et responsabilité est issue soit de la déverbalisation ou de la désadjectivation qui sont des règles de transformations syntaxiques, cet argument, pour faible qu'il est, ne tient pas pour la structuration interne de tels mots. Nous postulons, alors, que l'autonomie microsystémique est le droit linguistique fondamental de la morphologie car aucun microsystème de la langue ne peut revendiquer une autonomie totale. On ne peut pas dire que la syntaxe est totalement indépendante de la morphologie et de la sémantique. Dans la même vaine, ce serait impossible de postuler que la phonologie n'a rien à faire avec la phonétique et vice-versa. La sémantique, elle aussi ne peut se proclamer totalement indépendante des autres domaines de la linguistique.

\section{Références}

Anderson, Stephen R. (1982): "Where's Morphology?" Linguistic Inquiry 13: 571-612.

Aronoff, Mark (1976): Word Formation in Generative Grammar. Cambridge/London: MIT Press.

Asher, Ronald E./Simpson, J. M. Y. (eds.) (1994): The Encyclopedia of Language and Linguistics. Oxford: Pergamon Press.

Bach, Emmon (1983): "On the Relationship between Word-Grammar and Phrase-Grammar." Natural Language and linguistic theory 1: 65-90.

Beard, Robert (1995): Lexeme-Morpheme Base Morphology. New York: State University of New York Press.

Chomsky, Noam (1970): "Remarks on nominalizations." In: Jacobs, Roderick/Rosenbaum, Peter: (eds.) Readings in Transformational Grammar. Boston, Ginn: 111-176.

Chomsky, Noam (1981): Lectures on Government and Binding. Dordrecht: Foris Publications.

Corbin, Danielle (1987): Morphologie dérivationnelle et structuration du lexique. Paris: Niemeyer.

Di Sciullo, Anna-Maria/Williams, Edwin (1987): On the Definition of Word. Cambridge: MIT Press.

Dal, Georgette (1997): Un point de vue sur la morphologie dérivationnelle du français. http://www .mshs.univ-poitiers.fr/Forell/COLEX/DAL.DOC, consulté en mai, 2007.

Dell, François (1970): Les règles phonologiques tardives et la morphologie dérivationnelle du français. $\mathrm{PhD}$, MIT.

Fradin, Bernard (1994): "La théorie morphologique face à ses choix." Cahiers de lexicologie 63: 5-42.

Fradin, Bernard (2003): Nouvelles approches en morphologie. Paris: PUF.

Halle, Morris (1973): "Prolegomena to a theory of Word formation." Linguistic Inquiry 4: 3-16.

Halle, Morris/Marantz, Alec (1993): "Distributed Morphology and the Pieces of Inflection." In: Halle, Morris/Keyser, Samuel J. (eds.): The View from Building 20: Essays in Honour of Sylvain Bromberger. Cambridge, MIT Press: 111-176. 
Halle, Morris/Marantz, Alec (1994): "Some Key Features of Distributed Morphology." MIT Working Papers in Linguistics 21: 275-288.

Kerleroux, Françoise (2001): Les theories morphologiques à la fin $d u$ XXè siècle. http://infolang.u-paris10.fr/modyco/textes/Kerleroux/HSK-Kerleroux-2001.pdf, consulté en mai, 2007.

Kerleroux, Françoise (2006): "Les théories morphologiques à la fin du XX $\mathrm{X}^{\mathrm{e}}$ siècle." In: Auroux, Sylvain/Koerner, Ernst F. Konrad/Niederehe, Hans-Josef/Versteegh, Kees (eds): Manuel international sur l'évolution de l'étude du langage des origines à nos jours. Berlin, de Gruyter: 2313-2324.

Lapointe, Steven (1981): "The Representation of Inflectional Morphology within the Lexicon." North East Linguistic Conference 11: 190-204.

Perlmutter, David M. (1988): "The Split Morphology Hypothesis." In: Hammond, Michael/Nonnam Michael (eds): Theoretical Morphology: Approaches in Modern Linguistics. San Diego: Academic Press: 79-100.

Saussure, Ferdinand de (1995): Cours de linguistique générale. Paris: Éditions Payot \& Rivages.

Scalise, Sergio (1984): Generative Morphology. Dordrecht: Foris.

Selkirk, Elisabeth (1982): The Syntax of Words. Cambridge: MIT Press.

Zwicky, Arnold (1991): "Some Choices in the Theory of Morphology." In: Levine, Robert (ed.): Formal Grammar: Theory and Implementation. Vancouver, University of British Columbia: 327-371. 